\title{
Prevalência de síndrome metabólica em usuários de um ambulatório de avaliação perioperatória
}

\author{
Prevalence of metabolic syndrome in users of a perioperative outpatient clinic \\ Prevalencia del síndrome metabólico en usuarios de una consulta externa perioperatoria
}

Recebido: 13/01/2021 | Revisado: 15/01/2021 | Aceito: 19/01/2021 | Publicado: 24/01/2021

\author{
Rayla Caroline Carvalho Reis \\ ORCID: https://orcid.org/0000-0001-8884-8329 \\ Escola Superior de Ciências da Saúde, Brasil \\ E-mail: raylacaruline@gmail.com \\ Mirce Meire Gonçalves de Sousa Wilk \\ ORCID: https://orcid.org/0000-0001-6286-9631 \\ Fundação de Ensino e Pesquisa em Ciências da Saúde, Brasil \\ E-mail: mircemeire_wilk@hotmail.com \\ Sônia Maria Alves Gomes \\ ORCID: https://orcid.org/0000-0001-8178-860X \\ Fundação de Ensino e Pesquisa em Ciências da Saúde, Brasil \\ E-mail: sonia11.gomes@gmail.com \\ Suely Moreira do Nascimento Araujo \\ ORCID: https://orcid.org/0000-0002-9991-8115 \\ Fundação de Ensino e Pesquisa em Ciências da Saúde, Brasil \\ E-mail: suelymoreira2007@gmail.com \\ Francisca de Paula Carvalho Demes \\ ORCID: https://orcid.org/0000-0003-3927-4634 \\ Fundação de Ensino e Pesquisa em Ciências da Saúde, Brasil \\ E-mail: franciskadepaula@hotmail.com
}

\begin{abstract}
Resumo
Objetivou-se identificar a prevalência e fatores associados à Síndrome Metabólica em pacientes atendidos em um ambulatório multiprofissional de medicina e enfermagem perioperatória. Métodos: Trata-se de uma pesquisa descritiva, documental de natureza transversal, com abordagem quantitativa, realizada em prontuários de pacientes submetidos à avaliação pré-anestésica em um ambulatório de medicina e enfermagem perioperatória. Resultados: A prevalência de Síndrome Metabólica (SM) foi de 54,4\%, A população estudada caracterizou-se pelo predomínio de indivíduos do sexo feminino (67,6\%), a média de idade foi 62,36 anos com e desvio padrão de 13,69. Verificou-se associação significativamente, com a idade, IMC, SAHOS, HAS, DM e ASA Conclusão: Os parâmetros relacionados aos componentes da síndrome metabólica se mostraram aumentados para circunferência abdominal, PAS, PAD, GJ, e TG, e reduzidos para HDL entre os diagnosticados com SM. Ademais valores de IMC indicativos de sobrepeso e obesidade e presença de HAS e DM foram observados.
\end{abstract}

Palavras-chave: Enfermagem perioperatória; Síndrome metabólica; Hipertensão; Resistência à insulina; Obesidade abdominal.

\begin{abstract}
The aim of this study was to identify the prevalence and factors associated with Metabolic Syndrome in patients treated at a multiprofessional outpatient clinic for perioperative medicine and nursing. Methods: This is a descriptive, documentary cross-sectional study, with a quantitative approach, performed on the medical records of patients undergoing pre-anesthetic evaluation in a perioperative medicine and nursing clinic. Results: The prevalence of Metabolic Syndrome (MS) was 54.4\%. The studied population was characterized by the predominance of female individuals (67.6\%), the average age was 62.36 years with and standard deviation of 13.69 . There was a significant association with age, BMI, OSAHS, SAH, DM and ASA Conclusion: The parameters related to the components of the metabolic syndrome were increased for waist circumference, SBP, DBP, GJ, and TG, and reduced for HDL among those diagnosed with MS. In addition, BMI values indicative of overweight and obesity and the presence of SAH and DM were observed.
\end{abstract}

Keywords: Perioperative nursing; Metabolic syndrome; Hypertension; Insulin resistance; Obesity abdominal.

\section{Resumen}

El objetivo de este estudio fue identificar la prevalencia y factores asociados al Síndrome Metabólico en pacientes atendidos en una consulta externa multiprofesional de medicina perioperatoria y enfermería. Métodos: Se trata de un 
estudio descriptivo, documental, transversal, con abordaje cuantitativo, realizado sobre la historia clínica de pacientes sometidos a evaluación preanestésica en una clínica de medicina perioperatoria y enfermería. Resultados: La prevalencia de Síndrome Metabólico (SM) fue 54,4\%. La población estudiada se caracterizó por el predominio de individuos del sexo femenino (67,6\%), la edad promedio fue 62,36 años con desviación estándar de 13,69. Hubo asociación significativa con edad, IMC, SAHOS, HSA, DM y ASA Conclusión: Los parámetros relacionados con los componentes del síndrome metabólico se incrementaron para circunferencia de cintura, PAS, PAD, GJ y TG, y disminuyeron para HDL entre los diagnosticados con EM. Además, se observaron valores de IMC indicativos de sobrepeso y obesidad y la presencia de HSA y DM.

Palabras clave: Enfermería perioperatoria; Síndrome metabólico; Hipertensión; Resistencia a la insulina; Obesidad abdominal.

\section{Introdução}

Considerada como um problema de saúde pública, a síndrome metabólica (SM) é um termo que diz respeito a um conjunto de desordens cardiometabólicas, cujos fatores de risco presentes elevam as chances de surgimento de doenças cardíacas, derrames e diabetes. É caracterizada pela presença dos fatores, pressão arterial (PA), glicemia de jejum (GJ), triglicerídeos plasmáticos (TG-c), e circunferência abdominal (CA) elevados, além de níveis plasmáticos de HDL colesterol (High Density Lipoprotein-colesterol) reduzidos (Araújo et al., 2015; Mendes et al., 2012; Ministério Da Saúde, 2018).

Em termos diagnósticos a presença da SM é considerada quando há a existência de três ou mais desses fatores, de acordo com as seguintes referências de valores: CA maiores que $102 \mathrm{~cm}$ para homens e $88 \mathrm{~cm}$ para mulheres; níveis de HDL menores que $40 \mathrm{mg} / \mathrm{dl}$ para homens e $50 \mathrm{mg} / \mathrm{dl}$ para mulheres; níveis plasmáticos de TG igual ou superior a $150 \mathrm{mg} / \mathrm{dl}$; PA igual ou superior a 135/85 mmHg ou utilização de medicamentos anti-hipertensivos; e GJ com valor igual ou superior a $110 \mathrm{mg} / \mathrm{dl}$ (Cuppari, 2014; Sociedade Brasileira De Cardiologia, 2005).

No Brasil a SM se comporta com prevalência elevada e significativa, praticamente, em todas as faixas etárias, apresentando aumento, sobretudo, com o avançar da idade. Dados de pesquisas nacionais apontam prevalência de 63,9\% em idosos, 53,7\% em adultos, e 2,6\% em adolescentes, tendo como componentes de risco maior a circunferência abdominal aumentada, alterações nos níveis de pressão arterial, HDL-c baixo, e triglicerídeos elevados(Bortoletto et al., 2016; Kuschni et al., 2016; Pereira, Gomes \& Schwanke, 2016; Ramires et al., 2018).

Ao nível internacional estudos revelam também prevalência elevada de SM em diferentes faixas etárias, com predominância significativa no sexo feminino, em indivíduos com obesidade, e com idades maiores de 50 anos (ChimboYunga et al., 2017; Jmal et al., 2019; Marbou \& Kuete, 2019; Quiroz et al., 2018).

Desse modo e em virtude de sua alta prevalência e complexidade, a SM tem sido um fator de muita cautela no momento da avaliação pré-operatória, devido ao risco de complicações no peri e pós-operatório apresentado por aqueles que a contém. Complicações essas que aumentam a chance de mortalidade operatória e pós-operatória, recuperação mais lenta da função, pior prognóstico, piora da hiperglicemia em pacientes diabéticos, tempo prolongado de internação e alta em vários tipos de cirurgia, reduzindo assim a eficácia das intervenções cirúrgicas (Brackbill, Sytsma \& Sykes, 2009; Norris, Ralph \& Moloney, 2017; Pontes et al., 2017).

Portanto a avaliação pré-operatória precisa ser cuidadosa a fim de identificar pacientes com a SM. Todo paciente deve ser investigado em vista de situações específicas que possam interferir na anestesia e cirurgia. Dessa forma esse procedimento tem por objetivo realizar a identificação de pacientes em que o período perioperatório pode oferecer um risco maior de morbidade e mortalidade, bem como facilitar a elaboração de estratégias perioperatórias para a redução de riscos adicionais (Bierle et al., 2020; Hert et al., 2018).

Assim Shariq et al. (2018) evidenciaram associação entre a SM e aumento de complicações pós-operatórias, como elevação na necessidade de transfusão de sangue, além de utilização de recursos hospitalares em pacientes submetidos à adrenalectomia laparoscópica. Nessa perspectiva Chen et al. (2019) observaram aumento de mortalidade operatória em 
pacientes com SM submetidos à cirurgia de revascularização do miocárdio, o que coloca o paciente com essa condição em classificação cirúrgica de risco elevado.

Em face dos desfechos desfavoráveis apresentados no perioperatório, o estudo se faz relevante por apresentar a sociedade acadêmica e profissional, a prevalência de pacientes e dados clínicos e epidemiológicos de pacientes com a SM passíveis de cirurgia, havendo assim a oportunidade delinear estratégias de prevenção quanto às complicações.

\section{Metodologia}

Trata-se de uma pesquisa descritiva, documental de natureza transversal com abordagem quantitativa, foi desenvolvido conforme os requisitos propostos pela Resolução 466/2012 do Conselho Nacional de Saúde (CNS) (BRASIL, 2012), A pesquisadora responsável seguiu todos os preceitos bioéticos (autonomia, não maleficência, beneficência e justiça) contido nessa resolução e foi aprovado através com parecer 4.159.316, e Certificado de Apresentação para Apreciação Ética (CAAE) número 34229520.9.0000.5058, pelo Comitê de Ética e Pesquisa (CEP) da UNIPLAN ambos disponibilizados no parecer consubstanciado.

A pesquisa foi conduzida no período de maio a dezembro de 2020 e realizada em um Ambulatório de Medicina e Enfermagem Perioperatória (AMME) em um hospital público do Distrito Federal, Brasil. A população constituiu-se de 2160 prontuários de ambos os sexos, submetidos à consulta de avaliação pré-anestésica de alto risco no AMME, do período de junho de 2018 a junho de 2020. O tamanho amostral foi estimado por meio da fórmula para populações finitas para estudos transversais. Foram considerados como parâmetros o coeficiente de confiança de 95\% (1,96), erro tipo 1 de 5\%, o erro amostral de 5\%, proporção de ocorrência do fenômeno, como regra geral utilizou-se, $\mathrm{p}=50 \%$, pois não tinha informações sobre o valor esperado. Assim, a partir da aplicação da fórmula a amostra estimada foi em 327 prontuários e selecionados por amostragem aleatória simples.

As informações colhidas para o preenchimento dos formulários foram obtidas através de informações contidas nos prontuários eletrônicos dos pacientes. Foi utilizado um formulário estruturado e preenchido pela pesquisadora responsável, as variáveis do estudo foram divididas em: sociodemográfico, hábitos de vida, dados antropométricos, condições e avaliação clínica perioperatória, e exames laboratoriais. Não houve mecanismos diretos ou indiretos que colocaram em risco a integridade física dos participantes ou os prontuários dos mesmos. Todos os dados coletados foram resguardados com privacidade e usados somente para os fins a que se destina o estudo.

Para a classificação da SM os pacientes do estudo tinham que se enquadrar em três ou mais fatores alterados relacionados à SM, segundo a $\mathrm{SBC}$ nos qual: $\mathrm{CA}$ ( $\geq 102 \mathrm{~cm}$ para homens e $\geq 88 \mathrm{~cm}$ para mulheres), níveis de $\mathrm{HDL}$ ( $\leq 40 \mathrm{mg} / \mathrm{dl}$ para homens e $\leq 50 \mathrm{mg} / \mathrm{dl}$ para mulheres) níveis plasmáticos de TG $(\leq 150 \mathrm{mg} / \mathrm{dl})$, PA $(\leq 135 / 85 \mathrm{mmHg})$ ou utilização de medicamentos anti-hipertensivos e $\mathrm{GJ}(\leq 110 \mathrm{mg} / \mathrm{dl})$.

Os dados foram tabulados e analisados no Statistical Package for the Social Sciences (SPSS), versão 20.0. Foi realizada estatística analítica descritiva: frequência simples, desvio padrão, média. Na associação das proporções de variáveis, foi empregado teste do Qui-quadrado e foi adotado um intervalo de confiança de 95\% e nível de significância de 0,05.

Foram elaboradas tabelas, a fim de apresentar os resultados com detalhamento para melhor compreensão. Os resultados foram analisados e discutidos com base na literatura científica pertinente sobre a temática.

\section{Resultados}

A coleta de dados dos pacientes ocorreu no período de junho a novembro de 2020, apresentando um total de 327 pacientes para análise. A idade média de todos os pacientes foi de $62,36( \pm 13,6)$ anos com variação mínima de 28 a máxima de 92 anos. Outras características sociodemográficas e hábitos de vida reunidos na Tabela 1. 
Tabela 1 - Caracterização dos participantes da pesquisa segundo as varáveis sociodemográficas e hábitos de vida. Brasília DF, 2020. $(n=327)$.

\begin{tabular}{llll}
\hline Variáveis & $\boldsymbol{F}$ & \% & Média \pm DP \\
\hline Sexo & 106 & 32,4 & $62,36 \pm 13,6$ \\
Masculino & 221 & 67,6 & \\
Feminino & & 7,6 & \\
Idade & 25 & 41,6 \\
$18-39$ anos & 136 & 43,5 \\
$40-59$ anos & 142 & 7,3 \\
$60-79$ anos & 24 & \\
$>=80$ anos & & 19,6 \\
Tabagismo & 64 & 80,4 \\
Sim & 263 & 3,7 \\
Não & & 96,3 \\
Etilismo & 12 & 315 & \\
Sim & & \\
Não & &
\end{tabular}

Fonte: Dados da pesquisa.

A população estudada caracterizou-se pelo predomínio de indivíduos do sexo feminino (67,6\%). Concernente as variáveis de consumo de tabaco e álcool a maioria da população não fazem o uso dessas substâncias (80,4\% e 96,3\% respectivamente), porém houve uma amostra ainda significativa de 16,6\% de tabagistas.

Na Tabela 2 organizaram-se as características clínica e avaliação perioperatórios dos pacientes.

Tabela 2 - Caracterização dos participantes da pesquisa segundo as varáveis clínicas e perioperatória dos pacientes. Brasília$\mathrm{DF}-2020 .(\mathrm{n}=327)$.

\begin{tabular}{lll}
\hline Variáveis & F & \% \\
\hline Diabetes & 118 & 36,1 \\
Sim & 209 & 63,9 \\
Não & & 67,3 \\
Hipertensão & 220 & 32,7 \\
Sim & 107 & \\
Não & & 54,4 \\
Síndrome Metabólica & 178 & 45,6 \\
Sim & 149 & \\
Não & & \\
SAHOS & 112 & 34,3 \\
Alto risco & 82 & 25,1 \\
Risco intermediário & 133 & 40,6 \\
Baixo risco & & \\
Clínica cirúrgica & 163 & 49,8 \\
Geral & 92 & 28,1 \\
Ginecológica & 31 & 9,5 \\
Urológica & 17 & 5,2 \\
Mastológica & 13 & 4,0 \\
Ortopédica & 11 & 3,4 \\
Outras & & \\
\hline
\end{tabular}


Quanto às condições clínicas e de avaliação perioperatória, a maioria da amostra 63,9\%, não eram diagnosticadas com DM, apresentando um percentual considerável de diabéticos $36,1 \%$, enquanto $67,3 \%$ eram diagnosticados com HAS. A prevalência de SM foi 54,4\%. Referente ao SAHOS 40,6\% tem o baixo risco de desenvolver apneia do sono, $34,3 \%$ foram classificados por alto risco e $25,1 \%$ tem o risco intermediário, totalizando 59,4\% que apresentaram algum tipo de risco.

Em relação à clínica cirúrgica, 49,8\% dos pacientes iriam se submeter a cirurgia geral, 28,1\% a clínica ginecológica, 9,5\% a clínica urológica, 5,2\% a clínica mastológica, 4,0\% a clínica ortopédica e 3,4\% iriam submeter a outros tipos de clínica cirúrgica.

Analisou-se o IMC dos pacientes os adultos e idosos separadamente conforme recomendado pelo SISVAN, onde os adultos foram classificados em: Baixo Peso (IMC $<18,5$ ), Eutrófico (IMC $\geq 18,5 \mathrm{a}<25$ ), Sobrepeso (IMC $\geq 25 \mathrm{a}<30$ ) e Obesidade (IMC $\geq 30$ ). Os idosos foram classificados em: Baixo peso (IMC $<22 \mathrm{~kg} / \mathrm{m}^{2}$ ), peso adequado (IMC entre $22 \mathrm{~kg} / \mathrm{m}^{2}$ e $27 \mathrm{~kg} / \mathrm{m}^{2}$ ) e sobrepeso (IMC> $27 \mathrm{~kg} / \mathrm{m}^{2}$ ). O resultado foi apresentado na Tabela 3.

Tabela 3 - categorização do IMC expresso em percentuais, dos participantes da pesquisa. Brasília-DF - 2020. N.

\begin{tabular}{lccc}
\hline Variáveis & $\boldsymbol{F}$ & $\mathbf{\%}$ & Média $\pm \mathbf{D P}$ \\
\hline Adultos & 2 & 1,2 & $29,42 \pm 6,01$ \\
Desnutrição & 50 & 31,3 & \\
Eutrófico & 48 & 30,0 & \\
Sobrepeso & 60 & 37,5 & $26,87 \pm 4,97$ \\
Obesidade & & & \\
Idosos & 30 & 18,0 & \\
Desnutrição & 56 & 33,5 & \\
Eutrófico & 81 & 48,5 & \\
Sobrepeso & & & \\
\hline
\end{tabular}

Fonte: Dados da pesquisa.

Pôde-se perceber através da Tabela 3 que 67,5\% da amostra de adultos estiveram com excesso de peso desde 37,5\% foram classificados com obesidade e 30,0\% com sobrepeso, enquanto a maioria dos idosos 48,5\% se classificou sobrepeso de acordo com o IMC, seguidos pelos eutróficos, 31,3\% e 34,7\% respectivamente.

Quanto as variáveis dos componentes da síndrome metabólica segundo a SBC foram distribuídas na Tabela 4:

Tabela 4 - caracterização dos componentes da síndrome metabólica. Brasília-DF - 2020. (n=327).

\begin{tabular}{|c|c|c|c|c|}
\hline \multirow[t]{2}{*}{ Variáveis } & \multicolumn{4}{|c|}{$\begin{array}{c}\text { Síndrome metabólica } \\
\text { Média } \pm D P\end{array}$} \\
\hline & Sim & & Não & \\
\hline Sexo & $\begin{array}{l}\text { Masculino } \\
(n=55)\end{array}$ & $\begin{array}{l}\text { Feminino } \\
(n=123)\end{array}$ & $\begin{array}{l}\text { Masculino } \\
(n=53)\end{array}$ & $\begin{array}{l}\text { Feminino } \\
(\mathrm{n}=96)\end{array}$ \\
\hline Circunferência abdominal & $104,10 \pm 12,09$ & $99,85 \pm 13,04$ & $87,68 \pm 13,71$ & $86,41 \pm 8,70$ \\
\hline PAS & $146,49 \pm 20,89$ & $148,90 \pm 24,97$ & $128,57 \pm 14,41$ & $128,15 \pm 14,97$ \\
\hline PAD & $85,78 \pm 10,22$ & $84,02 \pm 11,46$ & $73,94 \pm 9,96$ & $75,79 \pm 9,78$ \\
\hline GJ & $136,18 \pm 61,95$ & $144,02 \pm 60,35$ & $93,84 \pm 15,13$ & $92,90 \pm 17,29$ \\
\hline Triglicerídeos & $218,61 \pm 46,27$ & $206,53 \pm 102,82$ & $113,11 \pm 30,61$ & $119,15 \pm 34,37$ \\
\hline HDL & $38,74 \pm 7,81$ & $41,78 \pm 8,13$ & $54,56 \pm 8,99$ & $57,79 \pm 9,36$ \\
\hline
\end{tabular}

Fonte: Dados da pesquisa. 
A média da circunferência abdominal foi de $104,10( \pm 12,09)$ para o sexo masculino e $99,85( \pm 13,04)$ para o sexo feminino. Referente à PAS e PAD obteve a média de $146,49( \pm 20,89)$ e $85,78( \pm 10,22)$ e $148,90( \pm 24,97)$ e $84,02( \pm 11,46)$ do sexo masculino e feminino respectivamente. Quanto aos exames bioquímicos às medias em relação da GJ, TG e HDL, nessa ordem foram: $136,18( \pm 61,95), 218,61( \pm 46,27)$ e $38,74( \pm 7,81)$ para os homens e $144,02( \pm 60,35) 206,53( \pm 102,82)$ e $41,78( \pm 8,13)$ para as mulheres. Para os pacientes que se enquadravam ao diagnóstico de SM.

Já para os pacientes que não se enquadravam no diagnóstico da SM, os resultados de seus componentes foram média de $87,68( \pm 13,71)$ e $86,41( \pm 8,70)$ de circunferência abdominal para o sexo masculino e feminino nesta ordem. A PAS e PAD alcançou a média de $128,57( \pm 14,41)$ e $73,94( \pm 9,96)$ nos homens e $128,15( \pm 14,97)$ e $75,79( \pm 9,78)$ nas mulheres. As médias encontradas nos exames bioquímicos de GJ, TG e HDL foram de 93,84( $\pm 15,13), 113,11( \pm 30,61)$ e $54,56( \pm 8,99)$ para o sexo masculino e $92,90( \pm 17,29), 119,15( \pm 34,37)$ e $57,59( \pm 9,36)$ para o sexo feminino.

$\mathrm{Na}$ Tabela 5 mostra uma associação das variáveis sociodemográficas e antropométricas, avaliação clínica perioperatória com síndrome metabólica.

Tabela 5 - Associação das variáveis do estudo com a SM. Brasília-DF - 2020. (n=327).

\begin{tabular}{lll}
\hline Variáveis & $\boldsymbol{X}^{\mathbf{2}}$ & $\mathbf{P}$ \\
\hline Sexo & 0,800 & 0,371 \\
Idade & 15,97 & 0,003 \\
Tabagismo & 0,55 & 0,815 \\
Etilismo & 0,819 & 0,366 \\
IMC & 74,07 & 0,000 \\
SAHOS & 176,10 & 0,000 \\
Clinica Cirúrgica & 6,34 & 0,386 \\
HAS & 18,64 & 0,000 \\
DM & 27,13 & 0,000 \\
MET & 6,67 & 0,036 \\
ASA & 58,01 & 0,000 \\
LEE & 5,44 & 0,066 \\
ACP & 1,68 & 0,431 \\
\hline
\end{tabular}

Fonte: Dados da pesquisa.

Considerando-se o valor de $\mathrm{p}<0,05$ para as hipóteses formuladas, ao realizar o teste de Qui- quadrado entre as variáveis acima, constatou-se associações significativa entre $\mathrm{SM}$ e as seguintes variáveis: Idade ( $\mathrm{p}=0,003)$, IMC ( $\mathrm{p}=0,000)$, $\operatorname{HAS}(\mathrm{p}=0,000), \operatorname{DM}(\mathrm{p}=0,000), \operatorname{SAHOS}(\mathrm{p}=0,000)$ e ASA $(\mathrm{p}=0,000)$. As demais variáveis não ocorreram nenhuma associação.

\section{Discussão}

Perante os dados dessa investigação referentes a questão sociodemográfica observa-se predomínio de indivíduos do sexo feminino $(67,6 \%)$ em relação ao masculino (32,4\%), com variação de idade entre 18 a 92 anos. Em consonância a tais resultados estudos realizados com pessoas submetidas a algum tipo de cirurgia bariátrica também evidenciaram predomínio do sexo feminino com percentuais de $82,43 \%, 86,6 \%$ e $91,8 \%$, com faixa de idade entre 18 a 60 anos, vale ressaltar que a população desses estudos foi menor quando comparado ao presente estudo o que pode explicar altos percentuais para o sexo prevalente (Brito et al., 2017; Nassif et al., 2009; Rêgo et al., 2017).

Quando observado as variáveis do consumo de tabaco e álcool tem-se que a grande maioria não faz uso dessas substâncias (80,4\% e 96,3\% respectivamente), no entanto é preciso notar que mesmo alto o percentual de não tabagistas ainda se observa um percentual significativo (19,6\%) de indivíduos que o pratica. Nessa perspectiva Ozkan et al. (2017) avaliando o 
efeito da síndrome metabólica em pacientes submetidos a cirurgia de revascularização do miocárdio encontraram em análise pré-operatória percentuais de tabagistas e etilistas maiores em comparação ao desse estudo com 50\% e 17,2\% respectivamente.

Dessa forma o incentivo a não utilização e abandono do álcool e cigarro é uma medida necessária, uma vez que, seus desfechos são desfavoráveis à saúde e envolvem além de agravos respiratórios, aumento na chance para o desenvolvimento da SM, já que esses elevam a concentração de gordura abdominal, reduzindo a sensibilidade insulínica e aumentando os níveis glicêmicos, acrescendo o risco para doenças cardiovasculares (Maccaronea, Lima \& Ferreira, 2017; Neto et al., 2018; Sociedade Brasileira de Cardiologia, 2019: Volp et al., 2012).

Comparando os efeitos do álcool com a prevalência da síndrome metabólica e seus componentes, Suliga et al. (2019) comprovaram que o consumo habitual de álcool esteve relacionado a maior risco de SM, hipertensão, aumento da concentração de glicose e obesidade abdominal em homens e maior risco de concentração anormal de glicose em mulheres. Portanto a prevenção para o surgimento de DCVs passa também pela precaução a hábitos etilista e tabagista.

Em se tratando das condições clínicas e de avaliação perioperatória constata-se que 67,3\% eram diagnosticados com HAS, ao passo que 36,1\% apresentaram DM, percentual menor em relação aos não diabéticos, porém significativo. Em referência ao risco de desenvolver apneia do sono de acordo com o SAHOS 59,3\% apresentaram risco sendo 34,2\% em alto risco e $25,1 \%$ em risco intermediário. Já em relação à cirurgia a qual os pacientes iriam ser submetidos $49,8 \%$ seriam submetidos à cirurgia geral, $28,1 \%$ ginecológica, 9,5\% urologia, 5,2\% ortopedia e 7,4\% outras.

Achados pré-operatórios em cirurgia bariátrica e de revascularização do miocárdio também mostraram uma maioria de pacientes diagnosticados com HAS (55,2\%, 56,8\% e 89,1\%), e valores distintos em relação ao diagnóstico de DM (32,83\%, 33,2\% e 67,2\%) (Brito et al., 2017; Chen et al., 2019; Özkan et al., 2017).

A presença de morbidades como a HAS e DM em pacientes submetidos a cirurgia eleva o risco de complicações no pré, trans e pós-operatório, por isso a importância de uma avaliação completa pré-cirurgia, a fim de identificar riscos pela presença dessas comorbidades. Estudos indicam que a presença de histórico de acidente vascular encefálico, doença arterial periférica e doença renal crônica associada a hipertensão elevam a gravidade clínica de pacientes submetidos à revascularização miocárdica cirúrgica. Ademais o estresse metabólico ocasionado pelo procedimento cirúrgico induz a um aumento da necessidade de insulina, podendo levar a descompensação e hiperglicemia em pacientes com DM (Colósimo et al., 2015; Pontes et al., 2018).

Ao realizar uma estratificação em adultos e idosos quanto ao IMC observa-se que entre os adultos a maioria apresentou excesso de peso (67,5\%) com 30\% em sobrepeso e 37,5\% em obesidade, o que não difere dos idosos com 71,1\% em estado de sobrepeso. Em concordância Mendes et al., (2016) também encontraram alto percentual de indivíduos em excesso de peso (70,6\%) na faixa de idade entre 19 e 68 anos quando analisaram dados de candidatos ao transplante de fígado.

Nesse contexto o IMC vem sendo utilizado como um importante marcador da condição clínica de pacientes com a síndrome metabólica em acompanhamentos ambulatoriais, com intuito de avaliar o risco associado ao excesso de peso, uma vez que, a literatura mostra maior tempo de cirurgia e maior prevalência de complicações cardiovasculares em pacientes com peso elevado (Araujo; Araújo; Bezerra, 2014; Nassif et al., 2009).

Para mais a obesidade apresenta-se como sendo o maior fator de risco para o desenvolvimento da síndrome da apneia obstrutiva do sono. Em relação a literatura Brito et al. (2017) encontraram valor inferior à do presente estudo com apenas 5\% dos pacientes submetidos à cirurgia bariátrica com essa condição.

De acordo com os dados da pesquisa a prevalência de SM no AMME foi de 54,4\% corroborando com o encontrado por Pimenta et al. (2007) quando comparou a morbimortalidade de pacientes com e sem síndrome metabólica submetidos à cirurgia de revascularização miocárdica, o estudo apontou uma prevalência de 69,2\% sendo maior entre as mulheres $(80,8 \%)$ em relação aos homens $(65,4 \%)$. 
São vários os riscos perioperatórios em pacientes com a SM destinados a algum tratamento cirúrgico como aumento da morbimortalidade, complicações cirúrgicas, tempos de intubação, internação em UTI e hospital estendidos e comprometimento da eficácia das intervenções cirúrgicas (Bardakçi et al., 2007; Brackbill, Sytsma \& Sykes, 2009; Norris, Ralph \& Moloney, 2017).

Nesse sentido Hu et al. (2017), Peng et al. (2017) e Ozkan et al. (2017) evidenciaram, respectivamente, que a presença da SM antes da cirurgia esteve associada a um risco 2,3 vezes maior de mortalidade por câncer gástrico, atuou como fator preditor independente significativo de mortalidade por carcinoma epidermóide de esôfago em homens, e associou-se a taxas de morbidade aumentadas no período pós-operatório imediato após cirurgia de revascularização do miocárdio.

A respeito das características dos componentes da síndrome metabólica observa-se que os parâmetros estão aumentados para os diagnosticados em relação aos não diagnosticados. As médias da circunferência abdominal, PAS, PAD, GJ, TG, HDL para os homens com a SM foram respectivamente $104,10 \mathrm{~cm}, 146,49 \mathrm{mmHg}, 85,78 \mathrm{mmHg}, 136,18 \mathrm{mg} / \mathrm{dl}$, $218,61 \mathrm{mg} / \mathrm{dl}$ e $38,74 \mathrm{mg} / \mathrm{dl}$ e para as mulheres os valores foram os seguintes $99,85 \mathrm{~cm}, 148,90 \mathrm{mmHg}, 84,02 \mathrm{mmHg}$, $144,02 \mathrm{mg} / \mathrm{dl} ; 206,53 \mathrm{mg} / \mathrm{dl} \mathrm{e} 41,78 \mathrm{mg} / \mathrm{dl}$.

Em comparação com a literatura quanto aos parâmetros clínico-antropométricos de indivíduos submetidos à cirurgia encontram-se também valores de glicemia de jejum, e triglicerídeos significativamente maiores em relação aos sem a SM (Chen et al., 2019).

Nassif et al. (2009) aponta valores de aproximadamente 100,75 mg/dL de glicose em ambos os grupos de pacientes obesos mórbidos com síndrome metabólica, submetidos a bypass gastrointestinal em Y de Roux. Já Brito et al. (2017) constataram valores baixos para HDL-colesterol (média de 41,21 mg/dl) e alto para LDL-colesterol (média de 172,73 mg/dl) na maioria dos pacientes submetidos à cirurgia bariátrica, além de TG $(187,33 \mathrm{mg} / \mathrm{d})$, PAS (140,68 mmHg), e PAD $(90,15$ $\mathrm{mmHg}$ ) aumentados assemelhando-se aos resultados expressos nesse estudo.

Em referência a associação entre a SM com a idade, IMC, SAHOS, HAS e DM foi significativa o que mostra mais uma vez que faixas de IMC maiores, acúmulo de gordura, níveis pressóricos maiores e de glicemia alterada aumentam os riscos à saúde e desfechos pós-operatórios ruins. Assim e em vista de uma maior segurança no manejo de pacientes com a SM eleitos a cirurgia destaca-se a necessidade da avaliação pré-operatória, a fim de verificar e quantificar os possíveis fatores de risco e tomar medidas para caso possível, corrigir ou prevenir as complicações relacionadas no pós-operatório (Watters, 2002; Machado, Martins \& Caramelli, 2004).

\section{Conclusão}

Os resultados do nosso estudo demonstram que prevalência de SM foi de 54,4\% estando essa associada, significativamente, com a idade, IMC, SAHOS, HAS, DM, e ASA . Os parâmetros relacionados aos componentes da síndrome metabólica se mostraram aumentados para circunferência abdominal, PAS, PAD, GJ, e TG, e reduzidos para HDL entre os diagnosticados com SM. Ademais valores de IMC indicativos de sobrepeso e obesidade e presença de HAS e DM foram observados.

Desse modo perante os desfechos desfavoráveis que a presença da SM expõe ao paciente que será submetido a cirurgia uma abordagem bem conduzida é primordial para se traçar estratégias que visem reduzir o risco de complicações perioperatória. Portanto, uma avaliação diferenciada da SM no pré-operatória por uma equipe multiprofissional como enfermeiros, nutricionistas, cirurgiões e anestesistas deve ser realizada em cada paciente, a fim de ser capaz de fornecer suporte adequado.

Ressalta-se também que a contribuição desse estudo se dá por apresentar ao meio acadêmico e profissional, dados que podem servir de referência para o desenvolvimento de estratégias que visem a prevenção e/ou redução de complicações trans e 
pós-operatórias em pacientes com essa condição. Para mais também serve como um alerta a sociedade, mostrando que a prevenção ao surgimento da mesma torna-se ainda mais necessária.

\section{Referências}

Araújo, M. F. M., Freitas, R. W. J. F., Lima, A. C. S., Pereira, D. C. R., Zanetti, M. L., \& Damasceno, M. M. C. (2015). Relation between sleep quality and metabolic syndrome among university students. Text Context Nursing. 24(2), 505-512.

Araujo, N. R., Araújo, R. A., \& Bezerra, S. M. M. S. (2014). Repercussão do sobrepeso e da obesidade no pós-operatório da cirurgia de revascularização miocárdica. Rev Esc Enferm USP. 48(2), 236-241.

Bardakçi, H., Demirdas, E., Bahar, I., Vural, K., Yay, K., Çiçekcioglu, F., Yavas, S., Kilzeltepe, U., \& Birincioglu, L. (2007). Metabolic syndrome and coronary artery bypass surgery. Turco J thorac Cardiovasc Surg, 15(3), 187-191.

Bierle, D. M., Raslau, D., Regan, D. W., Sundsted, K. K., \& Mauck, K. F. (2020). Preoperative Evaluation Before Noncardiac Surgery. Mayo Clin Proc. 95(4), 807-822.

Bortoletto, M. S. S., Souza, R. K. T., Cabrera, M. A. S., \& González, A. D. (2016). Síndrome metabólica, componentes e fatores associados em adultos de 40 anos ou mais de um município da Região Sul do Brasil. Cad. Saúde Colet. 24 (1), 32-40.

Brackbill, M. L., Sytsma, C. S., \& Sykes, K. (2009). Perioperative outcomes of coronary artery bypass grafting: effects of metabolic syndrome and patient's sex. Am J Crit Care. 18(5), 468-473.

Brasil. Ministério da Saúde. Conselho Nacional de Saúde. Comissão Nacional de Ética em Pesquisa, CONEP. Resolução $n^{\circ} 466 / 12$ sobre pesquisa envolvendo seres humanos. Brasília, 2012.

Brito, A. P. S., Araújo, G. B., Mainardi, C. R., Martins Neto, E. S., Centeno, D. M., \& Brito, M. V. H. (2017). Análise da síndrome metabólica e dados clínicoepidemiológicos de pacientes submetidos à cirurgia bariátrica. Pará Res Med J. 1 (4), 1-7.

Chen, S. Li, J. Li, Q. Qui, Z, Wu, X. e Chen,L. (2019). Metabolic syndrome increases operative mortality in patients with impaired left ventricular systolic function who undergo coronary artery bypass grafting: a retrospective observational study. BMC Cardiovasc Disord . 19(25), 2-9.

Chimbo-Yunga, J. M., Chuchuca-Cajamarca, J. A., \& Encalada-Torres, S. W. L. E. (2017). Síndrome metabólico y actividad física en adultos mayores de la sierra ecuatoriana. Rev Salud Pública.19(6), 754-759.

Colósimo, F. C. Sousa, A. G. Silva, G. S. Piotto, R. F., \&Pierin, A. M. G. (2015). Hipertensão arterial e fatores associados em pessoas submetidas à cirurgia de revascularização do miocárdio. Rev Esc Enferm USP. 49(2), 201-208.

Cuppari, L. Guia de nutrição: clínica no adulto. (3a ed.), Manole, 2014.

De Hert, S. (2018). Pre-operative evaluation of adults undergoing elective noncardiac surgery: Updated guideline from the European Society of Anaesthesiology. Eur J Anaesthesiol. 5(6), 407-465.

Hu, D. Peng, F. Lin, X. Chen, G. Zang, H. Liang, B. Ji, K. Lin, J. Chen, L-F. Zheng, X., \& Niu, W.(2017). Preoperative Metabolic Syndrome Is Predictive of Significant Gastric Cancer Mortality after Gastrectomy: The Fujian Prospective Investigation of Cancer (FIESTA) Study. EBioMedicine. 15, 73-80.

Jmal, L. Jmal, A. Abdennebi, M. Feki, M., \&Boukthir, S.(2019). Prevalence of metabolic syndrome in Tunisian overweight and obese children. Tunis Med. 97(1), 133-139.

Kuschnir, M. C. C. Bloch, K. V. Szklo, M. Klein, C. H. Barufaldi, L. A. Abreu, G. A. Schaan, B. Veiga, G. V. Silva, T. L. N., \& Vasconcellos, M. T. L. (2016). ERICA: prevalence of metabolic syndrome in Brazilian adolescentes. Rev Saúde Pública. 50(1), 1-11.

Lima Neto, J. C. G. Oliveira, J. F. S. F. Souza, M. A. Araújo, M. F. M. Damasceno, M. M. C., \& Freitas, R. W. J. F. (2018). Prevalência da síndrome metabólica e de seus componentes em pessoas com diabetes mellitus tipo 2. Texto Contexto Enferm. 27(3), 2-8.

Maccaronea, S. D. Lima, D. B., \& Ferreira, E. B. (2017). Rastreamento da síndrome metabólica e qualidade de vida dos diabéticos adscritos a uma unidade de estratégia de saúde da família em um município do sul de Minas Gerais. Rev Aten Saúde. 15(51), 98-105.

Machado, F. S. Martins, M. A., \& Caramelli, B. (2004). Perioperatório: procedimentos clínicos. Sarvier.

Marbou, W., \& Kuete, V. (2019). Prevalence of Metabolic Syndrome and Its Components in Bamboutos Division's Adults, West Region of Cameroon. Biomed Res Int. 30, 2-12.

Mendes, K. G. Theodoro, H. Rodrigues, D. A., \& Olinto, A. T. M. (2012). Prevalência de síndrome metabólica e seus componentes na transição menopáusica: uma revisão sistemática. Cad. Saúde Pública. 28(8), 1423-1437.

Mendes, K. D., Lopes, N. L., Fabbris, M. A., Castro-e-Silva Júnior, O., \& Galvão, C. M. (2016). Caracterização sociodemográfica e clínica de candidatos a transplante de fígado. Acta Paul Enferm. 29(2), 128-135.

Ministério Da Saúde. Síndrome Metabólica. Biblioteca Virtual em Saúde.2018. http://bvsms.saude.gov.br/dicas-em-saude/2610-sindrome-metabolica.

Nassif, P. A. N. Lopes, A. D. Lopes, G. L. Martins, P. R. Pedri, L. E. Varaschim, M., \& Bopp, D. S. (2009). Alterações nos parâmetros pré e pós-operatórios de pacientes com síndrome metabólica, submetidos a bypass gastrointestinal em y de roux. ABCD Arq Bras Cir Dig. 22(3), 165-170. 
Norris, P. Ralph, N., \& Moloney, C. (2017). Does metabolic syndrome predict surgical complications? A protocol for a systematic review and meta-analysis. Syst Rev. 6(115), 2-7.

Özkan, S. Demirtunç, R. Özdemir, F. Uğur, O. Balcı, A. Y. Kızılay, M. Kaplan, M., \& Yekeler, I. The effects of the metabolic syndrome on coronary artery bypass grafting surgery. Cardiovasc JAfr. $28(1), 48-53$.

Peng, F. Hu, D. Lin, X. Chen, G. Liang, B. Zhang, H. Dong, X. Lin, J. Zheng, X., \& Niu, W. (2017). Analysis of Preoperative Metabolic Risk Factors Affecting the Prognosis of Patients with Esophageal Squamous Cell Carcinoma: The Fujian Prospective Investigation of Cancer (FIESTA) Study. EBioMedicine. 16, 115-123.

Pereira, A. M. V. B. Gomes, I., \& Schwanke, C. H. A. (2016). Síndrome metabólica em idosos assistidos na atenção terciária à saúde em Curitiba, Paraná: prevalência e associação com saúde, capacidade funcional, estilo de vida e fatores demográficos e socioeconômicos. Sci Med. $26(3), 2-9$.

Pimenta, E. Passarelli Junior, O. Borelli, F. Sousa, M. G. Gun, C. Amato, V., Amodeo, C., \& Piegas, L. S. (2007). Síndrome Metabólica em Pacientes Submetidos à Cirurgia de Revascularização Miocárdica: Prevalência e Marcador de Morbi-mortalidade no Período Intra-Hospitalar e após 30 dias. Arq Bras Cardiol. 88(4), 413-417.

Pontes, J. P. J. Saramago, A. L. P. Vasconcelos, M. M., \& Batista, N. R. (2017). Manejo pré-operatório das medicações para tratamento do diabetes mellitus. Rev Med.27(2), 83-91.

Pontes, J. P. J. Mendes, F. F. Vasconcelos, M. M., \& Batista, N. R. (2018). Avaliação e manejo perioperatório de pacientes com diabetes melito. Um desafio para o anestesiologista. Rev Bras Anestesiol. 68(1), 75-86.

Quiroz, D. Quiroz, D. Bognanno, F. J., \& Marin, M. (2018). Prevalência de síndrome metabólico y factores de riesgo en la etnia kariña, estado bolívar, venezuel. Rev Cient Cienc Méd. 21(1), 7-13.

Ramires, E. K. N. M. Menezes, R. C. E. Silva, G. L. Santos, T. G. Marinho, P. M., \& Silveira, J. A. C. (2013). Prevalência e Fatores Associados com a Síndrome Metabólica na População Adulta Brasileira: Pesquisa Nacional de Saúde. Arq Bras Cardiol. 110(5), 455-466.

Rêgo, A. S., Zulin, A. Scolari, S. Marcon, S. S., \& Radovanovic, K. A. T. (2017). Análise das condições clínicas de pessoas obesas em período pré e pósoperatório de cirurgia bariátrica. Rev Col Bras Cir.44(2), 171-178.

Shariq, A. O. Fruth, K. M. Hanson, T. K. Cronin, P. A. Richards, M. L. Farley, D. R. Thompson, G. B. Habermann, E. B., \& McKenzie, T. J. (2018). Metabolic syndrome is associated with increased postoperative complications and use of hospital resources in patients undergoing laparoscopic adrenalectomy. Surgery,163(1), 167-175.

Sociedade Brasileira De Cardiologia. (2019). Atualização da Diretriz de Prevenção Cardiovascular da Sociedade Brasileira de Cardiologia -. Arq Bras Cardiol, 113 (4), 787-891.

Sociedade Brasileira De Cardiologia. I diretriz brasileira de diagnóstico e tratamento da síndrome metabólica. Arq. Bras. Cardiol 2005 84(1):3-28.

Suliga, E. Kozieł, D., Ciesla, E. Rebak, D. Głuszek-Osuch, M., \& Głuszek, S. (2019). Consumption of Alcoholic Beverages and the Prevalence of Metabolic Syndrome and Its Components. Nutrients. 11 (11), 2-14

Volp, A. C. P. Brito, C. J. Roas, A. F. C. M. Córdova, C., \& Ferreira, A. P. (2012). Estilo de vida e síndrome metabólica: exercício e tabagismo como moduladores da inflamação. J Health Sci Inst. 30(1), 68-73.

Watters, J. M. (2002). Surgery in the elderly. Can J Surg.45(2), 104-108. 